\title{
REGISTRO DE INFORMAÇÕES EM ENFERMAGEM NA CONCEPÇÃO DE ENFERMEIROS
}

Anna Karine Dantas de Souza ${ }^{1}$, Sérgio Ribeiro dos Santos ${ }^{2}$

\begin{abstract}
RESUMO: Estudo exploratório-descritivo, cujo objetivo é conhecer a concepção dos enfermeiros quanto aos prontuários eletrônico e manual e identificar aspectos positivos e negativos referentes ao uso desses registros nos hospitais da cidade de João Pessoa-PB. Como método para a coleta de dados utilizou-se a entrevista semiestruturada; a técnica de análise adotada foi o discurso do sujeito coletivo. Os resultados evidenciaram que as vantagens do registro eletrônico, em relação ao manual são imensas, embora a satisfação com o prontuário informatizado não seja total. Percebeu-se ainda que os enfermeiros não estão adaptados a essa tecnologia e necessitam de mais conhecimento. O prontuário manual apresenta desvantagens por conter registros ilegíveis, possibilitar perdas de informações e não permitir que mais de uma pessoa utilize-o ao mesmo tempo. Comparando as opiniões dos enfermeiros constatou-se que consideram vantajoso o registro eletrônico em relação ao manual, embora não dominem essa tecnologia.
\end{abstract}

PALAVRAS-CHAVE: Sistemas de informação; Sistemas computadorizados de registros médicos; Enfermagem.

\section{INFORMATION REGISTRY IN NURSING IN THE NURSES’CONCEPTION}

\begin{abstract}
This is an exploratory-descriptive study with the goal to know the nurses' conception about both electronic and handbook records, and to identify positive and negative aspects concerning the use of such records in hospitals of the city of João Pessoa-PB. As a method for data collection it was used the semi-structured interview. The analysis technique adopted was the discourse of the collective subject. The results showed that the benefits of electronic record on the handbook are immense, but the satisfaction with the computerized medical record is not complete. It was even noticed that nurses are not adapted to this technology and need more knowledge to use it. The handbook records presents disadvantages for contain illegible records, and possible loss of information does not allow more than one person to use it simultaneously. Comparing the views of nurses, the study found that they believe the electronic registration has an advantage if compared to the handbook, although they do not master this technology.
\end{abstract}

KEYWORDS: Information systems; Medical records systems, computadorized; Nursing.

\section{REGISTRO DE INFORMACIONES EN ENFERMERÍA EN LA CONCEPCION DE LOS ENFERMEROS}

RESUMEN: Estudio exploratorio-descriptivo, cuyo objetivo es conocer la concepción de los enfermeros en relación a los prontuarios electrónicos y manual, e identificar aspectos positivos y negativos referentes al uso de dichos registros en los hospitales de la ciudad de João Pessoa, PB. Como método para la recolección de datos fue utilizada la entrevista semiestructurada; la técnica de análisis adoptada fue el discurso del sujeto colectivo. Los resultados mostraron que las ventajas del registro electrónico, en relación al manual, son inmensas, aunque la satisfacción con el prontuario informatizado no sea total. Se percibió todavía que los enfermeros no están adaptados a esta tecnología y necesitan más conocimientos. El prontuario manual presenta desventajas por contener registros ilegibles, posibilitar pérdida de informaciones y no permitir que más de una persona utilice al mismo tiempo. Comparando las opiniones de los enfermeros fue constatado que consideran ventajoso el registro electrónico en relación al manual, aunque no dominen esta tecnología.

PALABRAS CLAVE: Sistemas de información; Sistemas historias clínicas informatizadas; Enfermería.

${ }^{1}$ Graduada em Enfermagem pela Universidade Federal da Paraíba-UFPB.

${ }^{2}$ Enfermeiro. Administrador. Doutor em Sociologia. Professor Associado do Departamento de Enfermagem da UFPB.

Autor correspondente:

Sérgio Ribeiro dos Santos

Rua David Luna, 117/207 - 58033-090 - João Pessoa-PB

Recebido: 06/08/08

E-mail: srsantos207@oi.com.br

Aprovado: 25/09/09 


\section{INTRODUÇÃO}

O enfermeiro em seu dia-a-dia trabalha com um grande volume de informações. Para facilitar seu manejo são desenvolvidos alguns sistemas de informação como auxílio às suas atividades, especialmente no uso do prontuário. Este pode ser definido como um conjunto de elementos ou componentes inter-relacionados que coletam, manipulam, armazenam e disseminam os dados e informações, favorecendo um mecanismo de feedback ${ }^{(1)}$. No ambiente hospitalar tem por objetivo suprir as necessidades operacionais dos diversos setores ou serviços, assim como proporcionar a adequada integração e interação entre os mesmos ${ }^{(2)}$.

Alguns sistemas de informação destinam-se a um melhor controle dos prontuários dos pacientes, sendo conhecido por prontuário eletrônico do paciente. Consiste em um repositório de dados clínicos e demográficos que centraliza as informações sobre a saúde do paciente, com segurança e sigilo. Por ser um método moderno de utilização de registro eletrônico, o sistema vem despertando a curiosidade e o interesse de profissionais usuários de prontuários de registro manual.

Temos observado que esses enfrentam dificuldades por nem sempre terem as informações sobre os pacientes de forma organizada e acessível. Por outro lado, também percebemos que alguns profissionais de enfermagem, os quais têm à sua disposição informações organizadas no prontuário eletrônico, ainda apresentam resistência ao seu uso.

Ao abordar a informatização no processo de enfermagem observamos hoje mudanças do processo manual para o informatizado; em ambos há vantagens e desvantagens. Nesse sentido, o Prontuário Eletrônico do Paciente-PEP tem por objetivo padronizar e organizar, de maneira concisa e sistematizada, os dados relativos a cada paciente, otimizando a assistência prestada. Com sua adoção, é possível a introdução rápida de dados, ajudando a reduzir erros e padronizando os planos de cuidados ${ }^{(3)}$, de modo que os maiores beneficiados por esse sistema sejam os profissionais de enfermagem, pois são responsáveis por mais de $50 \%$ das informações contidas ${ }^{(4)}$.

Apesar de todo o avanço tecnológico, ainda existem inúmeras instituições de saúde que utilizam o registro manual. Em face desse contexto, surgiram os seguintes questionamentos que nortearam este estudo: Qual a concepção dos enfermeiros quanto ao uso dos prontuários eletrônico e manual? Quais são os aspectos positivos e negativos apontados pelos enfermeiros na utilização do registro informatizado? Portanto, este estudo tem o objetivo de conhecer a concepção dos enfermeiros quanto ao uso dos prontuários eletrônico e manual e identificar os aspectos positivos e negativos referentes ao uso desses registros.

\section{MATERIAL E MÉTODO}

Trata-se de uma pesquisa exploratória com abordagem qualitativa. Nas pesquisas qualitativas o campo de investigação é o espaço ocupado pelas relações sociais com o objeto de estudo ${ }^{(5)}$. Teve como cenário os serviços de enfermagem de um hospital privado e outro público localizados no município de João Pessoa, Estado da Paraíba.

O projeto foi aprovado pelo Comitê de Ética em Pesquisa sob o protocolo n ${ }^{\circ}$ 039/2007, em 08/05/ 2007. De forma que foram observadas as normas éticas contempladas na Resolução no 196/96 do Conselho Nacional de Saúde ${ }^{(6)}$, principalmente no que diz respeito ao Consentimento Livre e Esclarecido dos participantes, garantindo-lhe a liberdade e autonomia de desistir a qualquer momento de sua participação no estudo, assim como ao anonimato e sigilo quando da publicação da pesquisa.

Os atores envolvidos foram enfermeiros assistenciais cujo quantitativo de participantes não foi estabelecido, a priori, por se tratar de um estudo qualitativo; optamos pela amostragem teórica até alcançar o nível de saturação. Assim, percebemos que os discursos de 20 enfermeiros nos apontavam a saturação dos dados, de forma que participaram 10 enfermeiros de uma instituição hospitalar privada e os demais de uma instituição hospitalar pública.

A escolha dos participantes ocorreu de forma aleatória, conforme a disponibilidade e acessibilidade aos enfermeiros. E entre as diferentes formas de entrevista, escolhemos a entrevista semiestruturada. Para a realização das entrevistas, agendadas previamente, inicialmente esclarecemos os propósitos do trabalho, o uso de gravador, conforme explicitado no termo de Consentimento Livre e Esclarecido, não tendo havido restrições. As entrevistas duraram em média trinta minutos e os entrevistados não demonstraram dificuldades em expressar suas opiniões a respeito das questões formuladas.

A coleta de dados ocorreu durante o mês de junho de 2007. Os dados foram analisados qualitativamente por meio da técnica de análise do 
discurso do sujeito coletivo. Esta é uma estratégia metodológica de análise utilizada em pesquisa qualitativa e consiste num conjunto de procedimentos de tabulação de dados discursivos, provenientes dos depoimentos dos participantes do estudo, a fim de extrair um discurso único que represente todos ${ }^{(7)}$.

Tendo como fundamento a teoria da representação social e seus pressupostos sociológicos, a técnica busca analisar o material verbal coletado, extraindo-se de cada um dos depoimentos as idéias centrais e ancoragens. Busca-se também identificar as correspondentes expressões-chave que compõem um ou vários discursos-síntese, denominados de discursos do sujeito coletivo. Nele faz-se a reunião de todas as possibilidades imaginárias (discurso uno, diferente e antagônico) oferecidas por uma dada cultura, num dado momento, para pensar um dado tema. Posteriormente, esse discurso coletivo global é fragmentado em vários discursos coletivos, com a finalidade de tornar a exposição mais compreensível.

\section{RESULTADOS E DISCUSSÃO}

Apresentamos inicialmente as perguntas do roteiro de entrevista com as respectivas idéias centrais, seguindo-se o discurso dos enfermeiros.

\section{Depoimentos de enfermeiros de um hospital público que utiliza prontuário manual}

Questão 1 - Quais as vantagens em se usar o registro manual para o seu trabalho?

Ideia central (1) - Facilidade de uso e acesso do prontuário

No manual não existe a necessidade do pessoal ser treinado com tecnologias avançadas ou ter o mínimo de domínio no uso de computadores; É mais fácil porque a gente pode levar para todo canto, consultar em todo canto, diferente do registro eletrônico que se precisaria do computador; É mais prático, você vai só relatar as informações que está adquirindo e tudo que você efetuou junto àquele paciente; A única vantagem é que as informações ficam guardadas, fica documentado aquilo que você está fazendo, sua assistência; É muito mais gravável porque está lá, não se dissipa não se estereotipa, não foge da escrita, fica para sempre.
Os enfermeiros que fazem uso exclusivo do prontuário manual apontaram a facilidade em manuseá-lo, sendo uma entre suas principais vantagens. Os profissionais da saúde não estão familiarizados com a tecnologia da informação, nem com sistemas de informação. Essa situação causa insegurança no uso do computador, bem como dificuldades no domínio do conteúdo da informática ${ }^{(4)}$.

Apesar das facilidades no uso do prontuário manual, não são raras as incorreções ou omissões de registros. Na maioria dos casos as falhas gramaticais e ortográficas, o registro das relações factuais e o emprego dos termos em geral podem provocar distorções nas informações. Os registros bem formulados refletem a qualidade do serviço prestado, sendo também um elemento essencial para os casos de compensação financeira e para fins ético-legais ${ }^{(8)}$.

Ideia central (2) - Formato do prontuário.

Por ele ser feito de forma unificada, você tem acesso às informações de outros profissionais $e$ isso facilita fazer uma maior intervenção da parte da enfermagem; Você pode obter uma evolução mais específica sobre os pacientes, ficando uma assistência mais pessoal.

O prontuário do paciente foi desenvolvido para garantir que os profissionais se lembrassem, de forma sistemática, dos fatos e eventos clínicos sobre cada indivíduo. Dessa forma, todos os profissionais envolvidos no processo de atenção à saúde podem ter acesso às informações do paciente. Na instituição onde o paciente está recebendo cuidados, o prontuário representa o mais importante veículo de comunicação entre os membros da equipe de saúde responsável pelo atendimento ${ }^{(9)}$. A extração da Idéia Central 2 mostra a importância da qualidade do trabalho em saúde para o paciente. Mas, isso só acontece quando existe uma equipe multiprofissional e uma eficiente comunicação na prestação de cuidados.

Ainda, de acordo com os discursos dos enfermeiros, o formato do prontuário manual propicia melhor utilização dos registros. Os impressos das instituições estudadas são identificados e separados de acordo com a função de cada profissional, trazem o espaço específico que deve ser preenchido por médicos, enfermeiros, pelo assistente social, nutricionistas, pelo fisioterapeuta e psicólogo. Além disso, contém espaço reservado para arquivar exames. 
Questão 2 - Quais as desvantagens em se usar o registro manual para o seu trabalho?

Ideia central (1) - Ilegibilidade dos registros

A letra não é tão legível para a gente ler, tem colegas que fazem com que adivinhemos o que ele escreve e isso é perigoso; Precisa que tenhamos mais consciência, que escrevamos melhor e com a letra melhor, praticando a gramática e a caligrafia.

Uma falha na compreensão de um registro, principalmente, de prescrição médica, pode trazer danos irreversíveis aos pacientes. Os sistemas de informação em enfermagem têm como metas facilitar a leitura da documentação, garantir maior confiabilidade e exatidão da informação, além da padronização na coleta de dados e no registro ${ }^{(10)}$.

As anotações de enfermagem devem ser compreendidas por todos os profissionais da saúde, para que seja possível dar continuidade ao tratamento do paciente; o registro dessas anotações é exigido pelo código de ética da profissão.

Os dados referentes à conduta, tratamento e evolução do paciente devem ser claros, concisos e completos, incluindo eventuais detalhes do atendimento; a escrita deve ser legível. Caso não consiga ler as anotações feitas no prontuário, o profissional deve pedir esclarecimento a quem as escreveu, pois adivinhar as palavras escritas por outra pessoa pode resultar em erros graves $^{(8)}$. Assim, a escrita no prontuário manual representa um ato de responsabilidade no conjunto das atribuições dos profissionais da saúde, sendo uma questão de consciência ética. Portanto, o registro manual, no que se refere à escrita, constitui um aspecto relevante do prontuário.

Ideia central (2) - Problemas de indisponibilidade do prontuário

Existem vários profissionais querendo ter acesso ao prontuário ao mesmo tempo para fazer seus registros. Acho que isso dificulta. Portanto, será uma grande desvantagem se o número de computadores for insuficiente; Se estiver num setor e precisar, ou se estiver, digamos, com um profissional e outro profissional precisar, não se consegue o uso do prontuário manual ao mesmo tempo.

Com o prontuário manual, o registro ou consulta no mesmo período de atuação de diversos profissionais não é possível. É comum observar, no hospital escola onde realizamos o estudo, alguns médicos deixarem o prontuário em locais diferentes do setor onde deveria ser arquivado. Porém, não se deve colocar a culpa apenas nos médicos, outros profissionais também cometem o erro de retirar o prontuário do acesso dos demais. Em hospitais escola o problema da indisponibilidade do prontuário é algo preocupante pois, além dos profissionais, os acadêmicos também necessitam pesquisar e fazer seus registros. Como não é possível a disponibilidade do prontuário ao mesmo tempo surgem conflitos entre funcionários e estudantes de diferentes cursos.

Ideia central (3) - Facilidade de perder informações

Quando os registros não são bem arquivados, você pode perdê-los com facilidade. Quando às vezes a gente precisa, o Serviço de Arquivo Médico e Estatística-SAME não localiza e a gente acaba tendo que abrir outro prontuário, o que eu acho ser uma dificuldade muito grande. No caso, se fosse utilizado o prontuário eletrônico, seria bem mais rápido localizar as informações; As folhas ficam muito soltas, se perdem, não existe muita organização, demanda tempo para procurar informações, perde-se exame, perde-se raio- $X e$ uma série de coisas.

Tem-se observado que os registros são realizados não só pelos enfermeiros, mas também por auxiliares de enfermagem. Por outro lado, há perdas no registro de informações na maioria dos hospitais. Some-se a isso a ocorrência de falhas nos mecanismos de armazenamento dos prontuários, gerando dificuldades quando se procura recuperar dados para fins de pesquisa e análise ${ }^{(10)}$.

Ainda observa-se a existência de folhas soltas devido à excessiva manipulação, possibilitando que informações importantes sejam extraviadas. Outro fator constatado é a falta de sistematização do registro manual. Ainda em 1920, na Clínica Mayo, em Minnesota nos Estados Unidos, houve um movimento para padronizar o conteúdo dos prontuários por meio da definição de um conjunto mínimo de dados que deveriam ser registrados. Esse conjunto mínimo de dados criou uma estrutura mais sistematizada de apresentação da informação médica que caracteriza o prontuário do paciente de hoje ${ }^{(9)}$. Entretanto, apesar 
de todos os esforços de padronização, o prontuário ainda contém uma série de queixas, resultantes de exames, análises, planos terapêuticos e achados clínicos de forma muitas vezes desordenada. Com isso, nem sempre é fácil obter uma informação clara e objetiva sobre a evolução do paciente ${ }^{(9)}$.

Questão 3 - Você gostaria de utilizar o prontuário eletrônico? Por quê?

Ideia central (1) - Desejo de utilizar o prontuário eletrônico

Gostaria, porque iria facilitar bastante e tem rapidez; Gostaria, por esta possibilidade de ver o paciente como um todo e iria ter essa acessibilidade no prontuário eletrônico; É sempre bom ter essa experiência; Gostaria, porque aqui, por ser uma instituição onde a gente está sempre recebendo o alunado, formando profissionais, iria facilitar muito devido à praticidade. Só que, para isso, deveria de ter toda aquela preparação, porque, no grupo, a gente tem aquele pessoal que se formou recentemente e que está tendo uma vivência boa, e tem também aquele pessoal mais antigo, o que seria uma dificuldade maior.

Estudo revela que os profissionais da enfermagem estão insatisfeitos com o sistema de registro manual, pois o consideram limitado e ultrapassado. Além disso, alegaram que os outros profissionais não lêem o que a enfermagem registra ${ }^{(4)}$. Assim, para os enfermeiros, o sistema manual de registro não atende a complexidade da assistência de enfermagem e é considerado desmotivante.

Conforme se percebe nos discursos, a insatisfação dos pesquisados com o sistema de registro manual expressa o desejo de desvendarem as inovações tecnológicas e os benefícios oferecidos pelo prontuário eletrônico. Busca-se melhorar o processo de trabalho e a gestão da assistência de enfermagem. Todavia, embora os enfermeiros entrevistados tenham manifestado o desejo de utilizar o prontuário eletrônico, expressam também insegurança e medo. Apesar disso, os discursos demonstram sua disposição em aprender a utilizar essa nova tecnologia.

Contudo, é possível acreditar na mudança do perfil das atividades do enfermeiro, tendo em vista maior conscientização quanto à evolução do conhecimento da ciência e tecnologia ${ }^{(11)}$. Atualmente, o enfermeiro percebe a necessidade, quase obrigatoriedade, de se atualizar, conectar-se com a evolução do saber e da técnica, para não ficar obsoleto, perdendo assim espaço e mercado.

\section{Depoimento de enfermeiros de um hospital privado onde se utiliza prontuário eletrônico}

Questão 1- Quais as vantagens em se usar o prontuário eletrônico?

Ideias centrais (1) - Organização, agilidade, praticidade, rapidez e legibilidade

Eu acho que é fácil o acesso, não tem perda de tempo e é bem mais prático; Dá para ver os dias anteriores com maior facilidade sem estar manuseando papel. Localiza mais fácil, você tem uma percepção mais rápida de tudo que está acontecendo no hospital através desse sistema; Mais rapidez, mais eficiência, é melhor; A tendência é a gente entrar mesmo na era da informática, pela questão da agilidade, praticidade, da comunicação mais rápida, mais ágil; Agiliza o serviço em todos os aspectos.

O prontuário eletrônico é uma estrutura eletrônica para manutenção e armazenamento de informações sobre o estado de saúde do indivíduo, assim como os cuidados recebidos ao longo da vida. Dessa forma, muitos benefícios podem ser obtidos com a utilização desse sistema de armazenamento ${ }^{(4,8-9,12)}$, e destacam-se os seguintes: Acesso remoto e simultâneo, ou seja, vários profissionais podem acessar um mesmo prontuário simultaneamente e de forma remota. Isso significa que há possibilidade de transmissão via Web; os médicos podem rever e editar os prontuários de seus pacientes a partir de qualquer lugar do mundo; Legibilidade, uma vez que os registros manuscritos são difíceis de ler na maioria das vezes. Os dados na tela ou mesmo impressos são muito mais fáceis de ler; Segurança de dados, por meio de um sistema bem projetado, com recursos de backup seguro e planos de desastres, garantindo melhor e de forma mais confiável que os dados não sofrerão danos e perdas; Confidencialidade dos dados do paciente - 0 acesso ao prontuário pode ser dado por níveis de direitos dos usuários e este acesso pode ser monitorado continuamente; Integração com outros sistemas de informação, uma vez que no formato eletrônico os dados do paciente podem ser integrados a outros 
sistemas de informação e bases de conhecimento, sendo armazenados localmente ou à distância; Assistência à pesquisa possibilitando que os dados estruturados possam facilitar estudos epidemiológicos. Os dados em texto-livre podem ser estudados por meio de uso de palavras-chave.

Todos esses benefícios certamente irão contribuir para a obtenção de melhores resultados dos tratamentos realizados e atendimento aos pacientes, com possível redução de custos e com otimização dos recursos.

Questão 2 - Quais as desvantagens em se usar o prontuário eletrônico?

Ideia central (1) - Demora na utilização, prejudicando a assistência

Ele atrasa muito a gente, a gente fica mais limitada por ficar na frente do computador ao invés de estar junto ao paciente fazendo uma assistência de enfermagem mais qualificada; Acho o sistema demorado, é extenso demais, são cinco páginas que temos que preencher diariamente. A gente evolui, mas muitas vezes não vai nem junto do paciente porque não dá nem tempo; Rouba o tempo que deveríamos estar prestando uma assistência direta ao paciente no apartamento, porque consome muito o tempo da gente. E se estiver cheio, são três horas na frente do computador. Acho um pouco repetitivo na evolução, onde a primeira deve ser a mais completa e depois uma coisa mais resumida.

A “demora” apontada na ideia central está associada, muitas vezes, à falta de conhecimento em tecnologia da informação. Nesse sentido, observa-se a necessidade de incluir nos programas curriculares disciplinas que preparem os graduandos em enfermagem para que possam ingressar no ambiente de trabalho em condições de usar a tecnologia.

É importante que todos os usuários do sistema e a diretoria da instituição de saúde estejam cientes dos benefícios que o prontuário eletrônico pode oferecer. Sem esses esclarecimentos, o usuário pode não vislumbrar todas as vantagens oferecidas pelo sistema, tornando-o ineficiente, incapaz de atender as suas necessidades ${ }^{(9)}$. Desta forma, percebe-se que a assistência ao paciente é prejudicada, não por deficiência do sistema informatizado, mas por causa do despreparo de profissionais para a utilização de tecnologia da informação. Por não saber utilizá-la, desperdiçam tempo e o contato direto com o paciente fica cada vez mais restrito.

Ideia central (2) - Existência de problemas técnicos

Eu vejo como desvantagens os problemas técnicos, onde você perde tudo e vai ter que fazer tudo de novo. É horrível ter que fazer tudo de novo; Acontece do sistema ficar fora do ar, então, todos têm que escrever a mão, mas isso é raro e as informações de antes do sistema falhar não se perdem; Quando o sistema dá pane, passa-se o dia preenchendo as informações no modo manual.

Os profissionais da saúde permanecem muito tempo na ilha de computadores, procurando solucionar problemas que, na realidade, são mais deles do que propriamente do programa. Normalmente, são dificuldades operacionais aos quais todos estão submetidos como, por exemplo, a queda do sistema, impressora que não funciona ou com o próprio computador. Os profissionais com pouca prática em sistemas informatizados encontram-se em situação difícil para manter uma assistência de qualidade aos pacientes sob sua responsabilidade. Nesses casos, utilizam como justificativa "problemas técnicos", que na verdade são entraves oriundos do despreparo em acompanhar a evolução da tecnologia ${ }^{(3)}$.

Conforme se pôde observar nas entrevistas, os problemas técnicos geram maiores consequências, quando os profissionais deixavam para fazer seus registros em um determinado momento, geralmente ao final do expediente. Assim, quando ocorrem alguns imprevistos, todas as informações são perdidas. A informação deve ser coletada no momento do cuidado, do contrário, o dado clínico e administrativo exigirá mais tempo e recurso para ser encontrado, registrado, recuperado e analisado. Caso o profissional deixe para registrar a informação no final do plantão comprometerá a qualidade do registro, de forma que muita informação útil e importante poderá ser perdida ou esquecida, além de duplicar o esforço ${ }^{(9)}$.

\section{Análise dos discursos dos enfermeiros}

Apesar do desejo dos enfermeiros em introduzir o uso de computadores no processo de enfermagem, observou-se que a satisfação com o registro manual ainda é considerável. Os enfermeiros afirmaram que o sistema manual representa facilidade no registro, 
utilização e manuseio dos dados. Porém, apontaram algumas desvantagens: ilegibilidade dos registros, indisponibilidade quando mais de uma pessoa quer utilizá-los ao mesmo tempo, além disso, facilita a perda de informações. Esses aspectos também foram confirmados na literatura ${ }^{(4,9,12)}$. Observa-se, desta forma, que os profissionais estão cientes das desvantagens, porém a totalidade dos entrevistados manifestou o desejo de utilizar o prontuário eletrônico.

Nas entrevistas realizadas no hospital privado que utiliza o prontuário eletrônico, os enfermeiros alegaram algumas vantagens do sistema, entre elas: organização, praticidade, agilidade, legibilidade e segurança. Como desvantagens, a grande maioria reconhece que a assistência é prejudicada pelo uso dos computadores, que requerem muito tempo dos profissionais no registro. Esses resultados reafirmam os estudos desenvolvidos sobre essa temática.

Os desafios para o desenvolvimento e a implantação do prontuário eletrônico são grandes, além de complexos. As informações, para fins puramente gerenciais, não representam de modo geral um desafio para a adoção do prontuário eletrônico. Todavia, a dificuldade está no registro, controle e recuperação das informações clínicas, bem como na natureza organizacional e na forma de trabalho convencional dos enfermeiros. Sistemas integrados pressupõem serviços e organizações integradas e exigem, principalmente, profissionais preparados. Logo, estes aspectos resultam, muitas vezes, na barreira crítica para o desenvolvimento e adoção de um prontuário eletrônico.

\section{CONSIDERAÇÕES FINAIS}

Com os resultados encontrados, por meio da comparação do registro manual com o eletrônico, é possível perceber as vantagens do uso do processo informatizado. Entretanto, os enfermeiros ainda consideram o prontuário manual uma ferramenta importante para registro de dados, apesar de suas limitações e deficiências.

Os enfermeiros precisam ter acesso à informação correta para desempenhar a grande variedade de intervenções envolvidas com o cuidado de enfermagem. As demandas administrativas e legais, o aumento do conhecimento, os avanços tecnológicos e as novas modalidades terapêuticas favorecem o surgimento de desafios. Esses exigem dos enfermeiros maior competência técnica, controle de qualidade e cuidado individualizado ao paciente, aumentando sistematicamente a necessidade de registro de todo o processo do cuidado.

Considera-se que a tendência da informática na saúde para a adoção do PEP é cada vez mais uma realidade. Porém, o investimento é grande, tanto do ponto de vista humano como financeiro e organizacional. O processo é longo e a integração dos profissionais será gradativa. Porém, se o interesse é a busca da qualidade no atendimento à saúde da população, esse investimento deve ser prioritário.

\section{REFERÊNCIAS}

1. Dalfovo O. Quem tem informação é mais competitivo. Blumenau: Acadêmica; 2002.

2. Silva MF, Neves RFA, Faig SMM, Teixeira SH. Tipos de sistemas de informação em saúde no ambiente hospitalar [texto na Internet]. s/d [acesso em 2007 Jun 10]; São Paulo: Universidade Federal de São Paulo Escola de Paulista de Medicina; Disponível: http:// www.virtual.epm.br/material/tis/curr-med/temas/med5/ med5t41999/sis/sub5.htm.

3. Lessa MC, Pinha TVB, Silva TT, Teixeira MLO, Castelo Branco EMS. Comunicação em enfermagem: modernização do sistema de informação hospitalar - a utilização do prontuário eletrônico. An. 8. Simp. Bras. Comun. Enferm. May. São Paulo [online]. 2002 [acesso em 2008 Mai 05]; Disponível: http://www.proceedings.s cielo.br/scielo.php?pid=MSC0000000052002000200038\& script=sci_arttext

4. Santos SR, Paula AFA, Lima JP. O enfermeiro e sua percepção sobre o sistema manual de registro no prontuário. Rev Latino-Am Enferm. 2003;11(1):80-87.

5. Minayo MCS. O desafio do conhecimento: pesquisa qualitativa em saúde. São Paulo: Hucitec; 2006.

6. Ministério da Saúde (BR). Conselho Nacional de Saúde. Resolução no ${ }^{\circ} 196$, de 10 de outubro 1996. Diretrizes e normas regulamentadoras de pesquisas envolvendo seres humanos. Brasília; 1996. 18p.

7. Lefèvre F, Lefèvre AMC. Discurso do sujeito coletivo: um novo enfoque em pesquisa qualitativa. Caxias do Sul: Educs; 2005.

8. Tanji S, Dahmer M, Oliveira SEM, Silva CMSLMD. A importância do registro no prontuário do paciente. Enferm Atual. 2004;Nov/Dez;(4):16-20.

9. Marin H, Massad E, Azevedo Neto RS. O prontuário eletrônico do paciente na assistência, informação e 
conhecimento médico. São Paulo: FMUSP/UNIFESP/ OPAS; 2003.

10. Marin HF. Informática em enfermagem. São Paulo: EPU; 1995.

11. Santos MS. A (in)satisfação do enfermeiro no trabalho: implicações para o gerenciamento das ações de enfermagem - aspectos teóricos [dissertação]. Ribeirão Preto (SP): Escola de Enfermagem de Ribeirão Preto; 1999.

12. Silva SL, Marques IR. Análise do registro de dados obstétricos em prontuários. Cogitare Enferm. 2007;Abr/ Jun;12(2):150-6. 